\title{
Filigrane
}

Écoutes psychothérapiques

\section{Rêver en psychanalyse : entre l'ogre et la princesse}

\section{Marie Claire Lanctôt Bélanger}

Volume 16, numéro 1, printemps 2007

Les hauts lieux et non-lieux du rêve I

URI : https://id.erudit.org/iderudit/016176ar

DOI : https://doi.org/10.7202/016176ar

Aller au sommaire du numéro

Éditeur(s)

Revue Santé mentale au Québec

ISSN

1192-1412 (imprimé)

1911-4656 (numérique)

Découvrir la revue

Citer cet article

Lanctôt Bélanger, M. C. (2007). Rêver en psychanalyse : entre l'ogre et la princesse. Filigrane, 16(1), 44-60. https://doi.org/10.7202/016176ar

\section{Résumé de l'article}

Évoquant des créateurs tels que Strindberg, Bergman, Stevenson, l'auteur se demande pourquoi les peintres, les cinéastes, les poètes, les musiciens et les auteurs écrivent-ils leurs rêves ? Sur quelle vérité le rêve se forme-t-il ? Puis, en travaillant deux rêves personnels, un rêve d'enfance apporté en analyse et un de fin de cure, les principales notions du rêve seront reprises pour illustrer comment le rêve se nourrit de pulsionnel et d'infantile. En association avec un élément d'un rêve, Marie Bonaparte est évoquée dans son difficile rapport au sexe féminin. Enfin, le texte se conclut sur le rêve comme pensée de la nuit et espace de transformation. 


\title{
Rêver en psychanalyse: entre l'ogre et la princesse*
}

\author{
marie claire lanctôt bélanger
}

\begin{abstract}
Évoquant des créateurs tels que Strindberg, Bergman, Stevenson, l'auteur se demande pourquoi les peintres, les cinéastes, les poètes, les musiciens et les auteurs écrivent-ils leurs rêves? Sur quelle vérité le rêve se forme-t-il? Puis, en travaillant deux rêves personnels, un rêve d'enfance apporté en analyse et un de fin de cure, les principales notions du rêve seront reprises pour illustrer comment le rêve se nourrit de pulsionnel et d'infantile. En association avec un élément d'un rêve, Marie Bonaparte est évoquée dans son difficile rapport au sexe féminin. Enfin, le texte se conclut sur le rêve comme pensée de la nuit et espace de transformation.
\end{abstract}

\begin{abstract}
' 'ai, depuis toujours, pratiqué la lecture. Très tôt, dans ma vie de petite fille, comme pour beaucoup d'autres enfants, la lecture est devenue ma survie; la lecture est devenue un havre, une fuite, bien sûr, mais surtout, un important lieu de survie psychique. Il m'est arrivé de lire avec boulimie; et parfois d'écrire presque autant; écrire et lire se sont rapidement soudés, telles des activités tangentes, réversibles. Elles me mèneront à ce texte hybride, certains diraient un texte métis, qui oscille entre la lecture, l'écriture, le plaisir de rêver et celui de déchiffrer. Le rêve n'est-il pas un texte vivant, caviardé, troublant, évanescent et même parfois insistant?
\end{abstract}

\section{Lire les rêves}

La lecture m'a fait connaître le thêâtre. À l'adolescence, j'ai tenté de séduire le théâtre et, en retour, il m'a séduite par ce mouvement de projeter sur une scène extérieure, en les amplifiant, les deuils, les catastrophes, les traumas, les vilaines histoires de familles et les rêveries si semblables à celles que nous vivons au quotidien. Aujourd'hui, le théâtre me conduit, grâce à des détours et à des amitiés, jusqu'à August Strindberg. De Strindberg, nous connaissons surtout Mademoiselle Julie. Peu de metteurs en scène ont osé s'attaquer aux autres morceaux de son théâtre qui est, selon l'expression qui convient à Artaud, grand amateur de Strindberg, un théâtre de la cruauté. Cruauté, guerres des sexes, meurtres psychiques, suggestions mortifères, scènes odieuses entre homme et femme, amour maternel étouffant et aliénant, paternité non reconnue ou déniée par la mère, toutes les facettes du drame humain se retrouvent, chez Strindberg, exposées de façon aigüe, crue, jusqu'à l'insupportable.

* Version modifiée d'une conférence donnée à la Société psychanalytique de Montréal, le 9 mars 2006. 
«Les personnages de Strindberg sont des émanations de sa propre psyché tourmentée et de sa vie déchirée. [...] Les personnages ont l'air de se cogner au hasard dans une sorte d'espace vide. [...] [Ses œuvres] appartiennent à un théâtre de l'esprit et travaillent au fond de nous, comme de la musique qu'on se rappelle.» (Stirner, 1993, 293-294.)

Les multiples visages de la haine - dont particulièrement la haine de soi alimentent ce théâtre, miroir de l'âme individuelle. On s'y reconnaît avec horreur. Il faut dire que Strindberg connaissait l'âme humaine. Il avait, comme Freud, suivi les enseignements de Charcot et admirait Meynert. Il savait ausculter et déchiqueter l'âme humaine se traitant lui-même comme objet d'examen, de vivisection (Weiss, 1962). Son écriture jaillit de l'intérieur comme un cri qui nous atteint directement en ouvrant des blessures que l'on aimerait voir cicatrisées.

En 1901, paraît, à Vienne, Psychopathologie de la vie quotidienne. Dans un ajout qu'il intégrera dans le corps de son texte, Freud, à qui l'on a parlé de Strindberg - qui est ce on, Lou Andréa Salomé, un autre collègue ou un patient ? - Freud, donc, écrit:

«Pour se conformer au précepte «gnô-ti sé-a-o-ton», «connaistoi toi-même», il faut commencer par l'étude de ses propres actes et omissions, apparemment accidentels.

De tous les poètes qui se sont prononcés sur les petits actes symptomatiques ou actes manqués, ou ont eu à s'en servir, il en est bien peu qui aient aussi bien entrevu leur nature cachée et éclairé aussi crûment les situations qu'ils provoquent que le fit Strindberg (dont le génie fut d'ailleurs aidé dans ce travail par son propre état psychique profondément pathologique.)»

Puis, reprenant un texte de Strindberg - La chambre gothique - que le $\mathrm{D}^{\mathrm{r}}$ Karl Weiss, de Vienne, cite dans le Journal international de psychanalyse, Freud souligne cette phrase de Strindberg:

«La force psychique de la haine est plus grande que nous ne le croyons.»

Et, avant de conclure, Freud ajoute :

«C'est ainsi que, dans le domaine des actes symptomatiques, l'observation psychanalytique doit également accorder la priorité aux poètes. Elle ne peut que répéter ce que ceux-ci ont dit depuis longtemps.» (269-271) 
... Et dont ils ne sont pas maîtres, pourrait-on ajouter.

À la même époque, en 1901, peu de temps après la parution de L'interprétation $d u$ rêve, Strindberg écrivait Le songe, grande pièce baroque qui illustre sa fascination pour cette étrange subversion de la pensée que représente le rêve. Chose étonnante, dès le début du Songe, par deux fois, Strindberg utilise l'association libre. Des objets - une porte devant laquelle l'officier attend son éternelle Victoria, puis, un aconit bleu - deviennent des éléments qui le font associer et retourner à l'enfance. Alors qu'à Vienne, Freud met au point, à partir de ses propres rêves, la méthode de l'association libre, Strindberg en fait l'usage dans $L e$ songe où se côtoient des visions d'enfer à la Jérôme Bosch et la beauté, la nécessaire beauté. Voulant comprendre l'énigme de l'univers et celle du bonheur humain, avec beaucoup de poésie et de force, Strindberg pointe du doigt et rejette les bien-pensants; comme le fit Freud ailleurs, il pose la question de la vérité : la vérité est-elle dangereuse ? Certains le disent. La philosophie le sait. La psychanalyse également: la vérité sur soi si elle n'est pas dangereuse est du moins difficile d'accès. Tout comme dans le théâtre de Strindberg, la séance d'analyse baigne dans cette ambivalence du vouloir savoir sur soi et dans ce trouble qui souvent aveugle ou fait fuir. Et c'est dans la lignée de Nietzsche, son contemporain qu'il admirait beaucoup et avec qui il échangera quelques lettres, que Strindberg, rêveur et poète, conclut Le songe: quand tout est jeté au feu, quand «la souffrance est une rédemption et la mort une délivrance», les fleurs sauront pousser sur les cendres. Et, peut-être, l'écriture poindre.

Strindberg écrivait-il ses rêves? Le cinéaste Igmar Bergman le croyait peutêtre, lui qui donne aussi beaucoup d'attention aux rêves. Compatriotes, ces deux grands créateurs que sont Strindberg et Bergman, semblent avoir des histoires relationnelles qui se ressemblent. Des histoires difficiles. Dans Les fraises sauvages, ce grand film de 1957, Bergman reprend un élément du début du Chemin de Damas de Strindberg. Bergman, avec d'infimes nuances, transforme le texte de Strindberg - une scène où, entrant dans la psyché du héros, l'Inconnu entend le son du tocsin et celui d'une marche funèbre - pour y voir se dérouler la vision de sa propre mort, la vision terrifiante de son propre cadavre, de son propre enterrement, l'image de sa propre disparition. Bergman reprend à vif le désarroi et l'angoisse de mort qui alimentent les cauchemars. L'on se rappelle peut-être le début de ce film: un vieux professeur, Isak Borg - qui porte, comme il arrive souvent dans les rêves, les mêmes initiales que Bergman — rêve:

«Il se promène dans une ville déserte, en ruine, voit une horloge sans aiguilles ainsi qu'une montre sans aiguilles : il n'y a plus de temps. Puis, dans un silence troué du son du glas, arrive une voiture noire tirée par des chevaux; la voiture bute sur un lampadaire, perd une roue, une grosse roue, et repart en échappant un cercueil qui s'ouvre en tombant. Un bras en ressort. Le professeur s'avance, le touche et voit alors qu'il s'agit de lui, 
d'une représentation de lui-même mort qui l'attire avec vigueur vers le cercueil.»

Cette auto-représentation de sa propre mort surgit, dans ce rêve désagréable, au soir d'une vie où le travail scientifique réussi et reconnu côtoie le bilan des relations humaines ratées, effilochées. Dans le film, ce rêve sera le prélude d'un long retour au passé, retour à l'enfance et à la première jeunesse du héros jusqu'à la réconciliation avec la vie et jusqu'à l'acceptation des choses «telles qu'elles sont». Ce n'était, somme toute, peut-être pas vraiment un cauchemar. Mais plutôt un rêve d'angoisse de mort qui, quand il survient, nous arrache du sommeil et ferme l'écran du rêve. Plus encore qu'au cinéma, la magie du théâtre, que ce soit celui de Strindberg ou celui d'autres auteurs, opère chaque fois que le rideau s'ouvre et que la scène s'éclaire. Cela évoque, pour moi, à chaque représentation, la magie du rêve. La scène du rêve c'est, comme le dit Stevenson, ce grand rêveur:

«Ce petit théâtre de l'esprit que nous gardons brillamment illuminé tout au long de la nuit une fois que les becs de gaz sont éteints et que l'obscurité et le sommeil règnent sans partage dans le reste du corps.» (Stevenson, 1888, 148)

Fin XIX siècle toujours, restons-y encore un moment, Robert Louis Stevenson écrit ses rêves. En 1898, dans une maison de rondins, à la frontière des États-Unis et du Québec, il écrit Un chapitre sur les rêves dans lequel il livre plus que son intérêt, sa passion même pour les rêves. Stevenson appelait ses rêves ses trésors, ses brownies. C'est à partir de ce théâtre intérieur, la réserve onirique de ses rêves, qu'il écrivit ses histoires fantastiques: Docteur Jekyll et Mr Hyde ou encore L'île au trésor. Il lui arrivait même de lire en rêve! Il se représentait lui-même tel un spectateur qui, du fond de sa loge, jouit d'un spectacle où se déroulent «de meilleures histoires que celles qu'il aurait pu élaborer lui-même.» Les rêves sont, pour Stevenson, plus créatifs que lui-même ne l'est en état de veille. Comme si ce n'était pas lui, le moi diurne, qui rêvait, mais plutôt, multipliés en lui, jouaient sur une scène interne, ceux qu'il appelait «les petites gens », «des acteurs et des inventeurs indépendants », des personnages grouillants, sans morale, plein d'imagination, des collaborateurs invisibles, des collaborateurs de l'ombre. L'écrivain Stevenson aurait été d'accord avec ce mot que l'on prête souvent à Freud: «Dépêchons-nous d'explorer l'inconscient avant qu'il ne se referme.»(Dora, 2005, 129)

Pourquoi les créateurs, peintres, cinéastes, poètes, musiciens et auteurs écrivent-ils leurs rêves? Nous entendons souvent des témoignages de cette nature: avec une grande fidélité, les gens écrivent leurs rêves, les scrutent, les racontent parfois, les utilisent, comme Stevenson, Strindberg ou Bergman, dans leurs créations. Ces rêveurs y cherchent-ils une vérité sur eux, sur leur vie psychique, sur leur perception du monde ? Quel sort donnent-ils à ces hôtes de la nuit, à ces 
hôtes mystérieux et troublants faits d'images et de mots? Stevenson dit précisément, avant que Freud ne le formule, puisqu'il est mort en 1894 : «Nous ne nous connaissons nous-mêmes que grâce à ces vaporeuses images du passé.» Parfois, ceux qui scrutent leurs propres rêves tentent d'y découvrir une vérité sur le monde, sur les autres. Ils oublient alors que le rêveur est celui qui écrit le texte du rêve, qui le met en scène et qui l'interprète également. Ils oublient que c'est dans l'enceinte close de leur univers psychique que la représentation se déroule et que s'y réalise, en secret, un désir, un souhait caché, venu de l'enfance. Ils ne veulent pas savoir qu'il s'agit uniquement d'eux-mêmes dans leurs rêves: l'ombilic du rêve est en eux bien qu'il leur échappe. Ils oublient que celui qui rêve et que ce dont ils rêvent, c'est eux; ils oublient que ce qui tricote l'énigme de ces pensées de la nuit repose sur les mouvements mystérieux et les processus complexes de l'inconscient et que tous les personnages mis en scène sont autant de représentations d'eux-mêmes. Représentations qui prennent parfois la forme d'intrusions dérangeantes, violatrices même, effets d'inquiétante étrangeté que l'on pourrait être tenté d'écarter mais qui réussissent souvent à fasciner par leur musique secrète.

\section{Rêver avant l'analyse : l'ogre}

Les hommes rêvaient avant Freud, bien sûr. Avec Freud, par ailleurs, «Un savoir inédit est en train de naître, bouleversant jusqu' aux critères mêmes de la scientificité. » (Dora, 2005, 84) Un savoir bouleversant aussi les théories de la mémoire, du fantasme, du souvenir, tissés et retissés par un enchaînement affectif. Un savoir soutenu par la précieuse exigence de l'association libre, cette démarche rêveuse, qui permet de tourner le dos à la conscience rationnelle, à l'entendement logique tout en «rendant son avenir au passé.» Un savoir qui bouleverse les petites théories sur soi que l'on porte en soi lorsque l'on s'adresse à un psychanalyste.

J'ai beaucoup rêvé avant de venir en analyse. J'y ai retrouvé, au tout début, deux rêves: d'une part, un rêve récurrent, et, d'autre part, un rêve dont l'intensité restait très vive quoiqu'il eût été rêvé vingt ans auparavant. Petite, je m'étais confectionné un carnet de rêves. Ce n'était pas un vrai cahier comme ceux que je me procurerai plus tard. Il fallait que ce carnet soit un peu bizarre, entre le cahier et le calepin. Un peu mal organisé, un peu comme un rêve, hybride. J'y écrivais mes rêves, sans rien y comprendre, parce qu'ils débordaient d'intensité et que le geste d'écrire les apaisait souvent. Ou du moins tentait-il de les apaiser. J'écrivais mes rêves et ce que j'appelais naïvement mes poèmes - le rêve n'est-il pas une poésie involontaire ${ }^{1}$ - pour donner forme à une trop forte intensité. Je faisais, depuis l'enfance, un rêve récurrent. Il ressemble, dans son tracé, avec quelques déformations et quelques restes diurnes particuliers, à beaucoup d'autres rêves d'enfants et d'adultes. La peur, la frayeur en sonne la sortie.

«Assise sur le balcon bas d'une maison, mon petit frère à mes côtés, je voyais venir de loin un horrible cannibale qui courait vers nous. Le cannibale allait nous dévorer. Je tentais de crier, de 
nous protéger, de me sauver. Quelquefois, il attaquait mon petit frère. La plupart du temps, je me réveillais tremblante, effrayée, avant que le cannibale ne se jette sur nous, rassasiée de terreur. »

J'ai longtemps cherché, en ce début d'analyse, à savoir qui était cet horrible cannibale. Pourquoi nous en voulait-il autant? Qui était cet autre? Alors, me montait dans l'âme cet impensé, cet impensable, cette souffrance incompréhensible. Et la peur. La scène se déroulait dans une petite ville près de Montréal, ville où je suis née. Petite ville hostile à ma famille où, par ailleurs, j'aimais me retrouver en quelques rares visites et où, parmi les cousins-cousines, j'apparaissais telle une étrangère venue de la grande ville. Beaucoup de mes rêves de début d'analyse m'y ramenèrent. Le roman familial de mes origines s'y ancrait. Les rêves $m$ 'aidaient à reconstruire cette histoire un peu rocailleuse de l'arrivée de mes parents à Montréal et celle de ma naissance. Se construire dans une histoire, se raconter, se dire, constitue le privilège des premières années de psychanalyse. Je m'y suis perdue dans les rues et les chemins de cette petite ville pendant mille rêves. J'ai visité mes grands-parents, j'ai habité les maisons d'enfance de mes parents pendant mille autres. Mais de cannibale, point de traces. Si j'y retrouvais la scène du rêve - la maison, le balcon — personne, là-bas, ne semblait particulièrement répondre à la description du personnage de l'ogre ou du cannibale. C'était un rêve sans parole. Mon cri d'impuissance et de terreur restait étouffé dans ma gorge. L'image seule régnait dans un mouvement quasi-cinématographique de l'avancée du cannibale vers moi et mon petit frère.

J'ai su, plus tard, l'importance du visuel dans le rêve et comment, dans cet abandon corporel, la motricité réduite au minimum donnait accès au visuel. Le rêve est une activité de pensée qui produit des formes, des images, des mots également. Mais il y prévalence du visuel. Pontalis explique ainsi la figurabilité du rêve:

«Le dormeur n'ayant plus à sa disposition ni le langage articulé ni la motricité, il ne lui reste pour parler et se mouvoir que les images. Il va donc dire par les images et se mouvoir dans leur succession. Il fait en somme de nécessité vertu.» (Pontalis, 1987, 234)

Les images sont à la fois la force et la faiblesse du rêve, parce que ces images s'effacent parfois : combien de fois ai-je voulu raconter ou me raconter un rêve et, comme les images de Pompéi s'estompant au moment de leur découverte, même en s'approchant lentement, celles du rêve s'effacèrent devant mes yeux ? Reste une sensation, une impression, un vague souvenir, un mot. Et si les mots sont parfois traités comme des objets dans le rêve — par exemple la triméthylamine du rêve de L'injection faite à Irma de Freud ou le Sarquintuié de Marie Bonaparte - la nécessaire figurabilité qui s'impose lors de la production des images du rêve a 
quand même besoin de la parole, besoin des mots pour se raconter, même à soimême. Le rêve transforme «l'invisible et le visible de l'inconscient en lisible.» (Pontalis, 2001, 237) Les mots servent de relais et de support aux images du rêve. La régression du rêve est-elle régie par le lien particulier qu'entretiendrait le refoulé avec le visuel? Le rêve pourrait le laisser croire. Souvent avons-nous entendu un rêveur racontant son rêve parler «de son film», soulignant ainsi, sans le savoir, la prégnance du visuel. Et aussi, parfois, en même temps, la mise à distance du rêve: ce n'est qu'un film! Le puits du rêve est un miroir où le rêveur ne veut pas toujours se reconnaître.

Le rêve, davantage que le lapsus, l'acte manqué ou le symptôme, donne une présentation, une représentation visuelle du retour du refoulé. Lui seul donne cet accès visuel à l'intérieur de soi, accès à cette scène psychique individuelle sur laquelle se déroulent nos rêves. L'écran du rêve, dont parlait Bertram Lewin, donne à penser que la figurabilité entretient un lien particulier avec l'inconscient. Et quelquefois, le travail du rêve s'allie avec celui de l'art: certains rêves sont esthétiques; ils reprennent des images, des formes qui illustrent l'économie plastique du rêve ou celle du rêveur. Ces rêves puisent dans l'iconographie personnelle du rêveur à même les tableaux qui meublent son musée intérieur; ils expriment parfois des désirs de réalisation artistique, des désirs de création plus grands que nature.

Mon cannibale, venu de l'enfance, était bien laid. Je le vois encore derrière mes paupières. J'en ai longtemps cherché l'identité. La menace de dévoration dont il se montrait capable me restait énigmatique. Quelque chose du rapport premier à la mère s'y dessinait-il ? Malgré son masque masculin, le cannibale reprenait-il, sous son déguisement, la peur d'être dévorée par une mère ogresse ? J'étais chanceuse d'apporter ce rêve à mon psychanalyste. Ce rêve occupait, à côté de tous ceux qui se présentaient, la nuit, une lumière, une couleur, particulière. Il venait de loin. Il me ramenait en arrière, dans des lieux infantiles proches de la bouche d'ombre. J'ai pu retracer que ce rêve avait été fait, une première fois, vers l'âge de cinq ans. C'est l'écart d'âge qui me sépare de ce petit frère représenté dans le rêve. Un autre rêve survenu au moment où, précisément, je travaillais ce rêve plus ancien m'aida à en souligner la dimension violente. Le même petit frère s'y retrouvait. Mais, peut-être n'était-il pas seul, cette fois. Ou plutôt, je découvris qu'il pouvait en représenter d'autres, en condenser, en cacher d'autres. Le danger s'étendait à plusieurs enfants et gardait toute son opacité. Et sa terreur. J'ai dit que j'étais chanceuse d'apporter un rêve ancien, un fragment vivide d'infantile en analyse ; mais j'étais surtout chanceuse de travailler avec un psychanalyste qui aimait les rêves. J'imagine, j'aime imaginer qu'il devait être un grand rêveur lui-même. Je lui ai inventé une généalogie qui lui donnait un analyste prestigieux dont je lisais les écrits sur les rêves. Je crois qu'autant la capacité de rêverie de la mère aide l'enfant à se créer un espace psychique où son monde intérieur peut se déployer dans tous ses coloris, autant la précieuse capacité de rêver de l'analyste aide ses patients à rêver et à associer librement. Il ne s'agit pas, comme on pourrait le dire 
précipitamment avec envie, seulement de suggestion, ni d'incitation à rêver pour faire plaisir à l'analyste et ainsi se créer un faux self de bon analysant. La valeur apportée, au cours d'une analyse, au rêve et aux associations est garante de la valeur accordée à l'univers intérieur, à l'univers secret du sujet. Mes rêves m'aidaient à sortir d'une langue qui aurait pu s'obsessionnaliser ou se réfugier dans la théorie loin des affects et des égarements associatifs nécessaires au processus analytique.

J'ai appris, en analyse, à regarder le rêve comme un rébus, à le lire, à le déconstruire et à abandonner l'interprétation symbolique que j'avais coutume, comme beaucoup d'autres, de pratiquer. Les formules habituelles : "ce rêve veut dire», «ce rêve signifie » qui tentent globalement de cerner le sens glissent sur la surface du rêve et refusent de pénétrer son mystère. Si je sais d'emblée, dès le récit terminé, ce que signifie le rêve, alors ce n'est pas vraiment un rêve: pourquoi le désir refoulé aurait-il subi tant de travail, tant de transformation s'il est si facilement reconnaissable et saisissable dans une formule, fut-elle belle, au sortir du récit du rêve? Seul le fractionnement du rêve en petites unités pour associer laisse apercevoir le souhait qui s'y réalise et laisse pressentir l'œuvre de transformation qui dut se faire pour déjouer la censure. Il faut détisser fil-à-fil ce que le rêve, Pénélope de la nuit, a finement tissé et caché en son sein. Je savais tout cela, pouvais-je dire. J'avais lu L'interprétation des rêves. Freud avait, rappelle Didier Anzieu (1988, 34), pris dans un livre d'un psychiatre allemand, Griesinger, paru en 1861, l'idée que les «rêves et les psychoses sont accomplissement de désir». J'avais vaguement conscience d'un désir qui surgissait, tapi, au coin des diverses avenues du démembrement du rébus. Mais souvent, très souvent, je ne voyais rien. Il me fallait l'aide de l'analyste pour éclairer une scène parfois trop lumineuse, parfois trop sombre qui me laissait aveuglée et muette. Ainsi la petite ville du rêve, puis la maison, le balcon qui s'apparentait aussi à la maison de ma petite enfance, la lueur crépusculaire qui teintait le rêve, la multitude de frères et sœurs qui se cachaient sous le petit enfant unique assis à mes côtés, me confrontaient toujours à ce cannibale, cet ogre sorti tout droit des contes que, déjà, je lisais à l'époque.

$\mathrm{Au}$ moment où j'écris ces mots, une patiente m'a raconté un rêve dans lequel se chevauchent des écritures étrangères. Une de ces écritures est faite de caractères chinois. En l'écoutant, j'ai été prise d'un bref vertige et ramenée à l'enfance, ramenée précisément à ce rêve: dans ses versions récurrentes, le cannibale est devenu un chinois. S'y condensaient de multiples petites phobies que l'école savait alimenter autour de ce qui se nommait, alors, le «péril jaune». La couleur du rêve, sa pigmentation particulière - beaucoup de rêves se dessinent sur le jaune de l'horizon ou ont cette couleur ocre au fond de l'air ${ }^{2}$ - m'est revenue avec ce vertige. Combien de temps ai-je mis avant de reconnaître le cannibale en moi ? Combien de temps ai-je résisté à cette interprétation qui me faisait dévorer mon frère, le faisait disparaître dans la gueule de l'ogre. Longtemps. En passant par la phobie, il me semble que j'ai trouvé lentement, très lentement, que l'objet dont on a peur est aussi celui de son désir. Ou plutôt que c'est de son propre désir dont on 
a peur. Je pouvais jouer au Petit Poucet et sauver mes frères de l'abandon dans lequel nous laissaient nos parents. Je pouvais croire que j'étais celle qui allait les protéger de l'ogre et de l'abandon. Mais la violence de la scène me ramenait abruptement à la demande exclusive d'amour qui caractérise l'enfant. À l'avidité. Vouloir être aimée, demander à être choisie, désirer être la préférée, d'une part, puis, souffrir d'être rejetée, menacée de devoir quitter la maison et d'être envoyée, dans la petite ville éloignée, en pension chez mes grands-parents, d'autre part, sont les affects qui nourrissent le rêve.

Pour réaliser ces puissants désirs, pour contrer la détresse, il me fallait éliminer l'enfant neuf, celui qui, arrivant, délogeait tous les autres et devenait si important, si choyé par la mère: voilà quelques-uns des mauvais sentiments dans lequel baigne le rêve. J'ai appris, depuis, à ne pas en avoir honte. À les reconnaître, même sous leur déguisement et leur inversion, à en voir la trace effrayante dans les rêves et dans les fantasmes. À apprendre que le monde intérieur de l'espace du rêve n'est pas celui de l'agir externe. Qu'il y a séparation et frontières entre le dedans et le dehors. Et surtout que la machine à rêver et à fantasmer est sans limites: «Comme le rêve, l'analyse ouvre à l'illimité et l'apprivoise.» (Pontalis, 1990, 54) Le rêve participe à la conquête de la liberté psychique qu'offre la psychanalyse. Comme l'écriture, parfois. J'ai appris, à partir de mes propres rêves, que les rêves ne sont pas l'expression de bons sentiments, mais qu'ils sont construits à même le pulsionnel le plus primitif, le plus refoulé, le plus sombre de notre âme. Qu'il sont d'amour et de haine intenses, de barbarie et d'amoralité. Les rêves, pensées sauvages, sont projetés sur l'écran lumineux de nos nuits, «ce petit théâtre de l'esprit», «cet écran blanc de nos nuits noires » où le fil de la mémoire fait s'agiter avec fièvre des personnages dont la rencontre est si importante pour trouver un peu qui nous sommes vraiment.

Depuis, l'ogre a disparu, mais la petite ville où se situait le rêve reste encore marquée d'un coefficient négatif : il m'arrive encore d'y rêver et de m'y perdre, ou de devoir y retourner à mon corps défendant. Les vieilles et tenaces histoires de famille mêlées à celle de mon origine prennent encore le chemin du rêve. Réveillées par un événement de la veille, leur toxicité reste susceptible de devoir passer et repasser encore par l'atelier du rêve pour se transformer et s'apaiser puisque le rêve :

«[...] sert d'usine de traitement pour les surplus pulsionnels et de fantasmes du Moi vigile, et par conséquent allège ce dernier de tout ce qui ne peut, en lui, être suffisamment lié par la réalité.» (Guillaumin, 1979, 49)

\section{L'espace du rêve}

Rêver et travailler ses rêves permet de créer ce que Masud Khan appelle (1987), sous le modèle de l'espace transitionnel de Winnicott, l'espace du rêve. Un espace à utiliser, un espace psychique qui s'ouvre et se multiplie; un espace qui diffère de 
ce que l'on nomme les processus du rêve et les contenus : un espace à trouver et même à conquérir. Je crois que seule la création de cet espace du rêve donne accès à l'expérience du rêve. Le travail de la cure permet cette conquête, cette ouverture psychique qui conduit à une personnalisation de l'expérience du rêve et «limite l'acting out des rêves dans l'espace social ». Le rêve n'est pas un agir; il protège des agirs inconscients qui pourraient échapper au moi vigile. Rêver de meurtre n'est pas un meurtre et ne fait pas de soi un meurtrier mais permet d'illuminer avec beaucoup de liberté la scène inconsciente où se débat l'enfant. L'expérience du rêve, en elle-même, est expérience de satisfaction. Non seulement parce que le rêve est le gardien du sommeil, comme le sommeil est gardien du rêve, mais aussi, parce qu'en plus de la réalisation secrète d'un souhait, reste, intense, le plaisir de rêver. L'insomniaque se voit privé de rêver en même temps que de dormir. Et celui qui a peur de rêver s'empêche parfois de dormir pour ne pas utiliser sa capacité de rêver ou ne pas utiliser cet espace du rêve dont il craint les habitants nocturnes. Il est des moments où l'intensité des rêves trouble le sommeil. D'autres où l'écran $d u$ rêve (Lewin, 1936) est troué et ne réussit pas à garder les images.

Je me rappelle un patient, un jeune patient, un peu fauve par sa férocité et les addictions; il disait de lui : «J'ai été mangé, par en dedans, quelque part.»On retrouvait, chez lui, comme dans mon rêve, le cannibale et même l'auto-cannibalisme. L'écran de ses rêves était formé d'une surface noire dont les contours n'étaient pas définis, évoquant, selon lui, certaines émissions de télévision pour enfants - Sol et Gobelet, par exemple — dont les décors étaient construits comme des lieux mal délimités: la porte était suspendue dans l'espace, la fenêtre ouvrait sur un précipice, les objets reposaient mal sur le sol. Le fond noir ne garantissait aucune séparation entre le dedans et le dehors, aucune stabilité dans les murs, les toits et les planchers. Ce jeune homme rêvait beaucoup, des rêves crus, de meurtres sanglants, de poursuites, de batailles et de dévoration; rêves de King Kong, de requins, de vipères, montrant ainsi une psyché laissée sans protection aux drames et aux terreurs infantiles. Il rêvait même de son suicide. Je n'ai pas pu, pendant des années, m'approcher de ses rêves, ni les interpréter, ni encore moins en relever les éléments transférentiels très marqués. Il déposait ses rêves, petites créations de la nuit, échappées, paquets ficelés, dont il traînait le bruit troublant tout le jour durant, jusqu'à la prochaine séance. J'en notais la fonction dans le transfert plus que les contenus invariablement violents. S'il rêvait d'abord pour rêver, si ses rêves étaient sa chose, son territoire que, tel un carnassier, il dessinait et protégeait, il gagnait, malgré tout, petit à petit le plaisir de rêver et celui de me raconter ses rêves.

L'expérience du transfert — l'agir du transfert (Gribinski, 2005) — se nomme difficilement, se devine plus qu'elle ne se parle; le rêve, par ailleurs, se raconte et crée, par son récit, un lien, un nouvel espace. De sorte que l'expérience du rêve devenait, pour mon patient, une expérience de pensée vivifiante, valorisante. Peu à peu, il s'amusait à contempler, au réveil, ce qui s'était échappé de lui et qui, reprenant son histoire infantile, transformait son regard sur lui et sur sa vie intérieure. Il lui arrivait, comme à d'autres d'ailleurs, d'avoir hâte de se mettre au 
lit pour tenter de retrouver son rêve, l'excitation de son rêve, de le continuer, et surtout de répéter le plaisir éprouvé à créer un lieu qui se transformait à son insu et qui, lentement, commençait à avoir des contours, des limites, des frontières, de la constance. Avec lui, j'ai appris la patience. Avec lui, j'ai compris l'envie qu'il éprouvait pour l'analyste qui associait trop, trop vite pour lui ; avec lui, aussi, $\mathrm{j}$ ' ai appris la distance: le trop loin de l'abandon, le trop proche de l'intrusion. Il rêvait parfois qu'il attaquait quelqu'un, à la tête, par derrière, avec une hache. Qui attaquait qui ? Qui tentait de briser les liens? Son âme à vif et son besoin d'addiction se sont peu à peu calmés. Je crois que la création de l'espace du rêve et du plaisir de rêver y sont pour beaucoup puisque les rêves sont non seulement les témoins de la vie psychique mais qu'ils sont aussi de puissants agents de transformation psychique. Les rêves, le plaisir de rêver et de raconter permettent, dans la cure, la lente appropriation de son histoire et de sa vie intérieure. Ainsi, la maison psychique qu'il habitait en rêve, à la fin du travail avec moi, s'était agrandie, enrichie. Les murs avaient une consistance et une délimitation plus précises, moins floues. Quelques trésors se cachaient dans les pièces. La porte en était accessible, les fenêtres s'ouvraient, annonçant la fin de la cure.

\section{Rêver dans la cure : la princesse}

D'autres rêves de fin d'analyse gardent jalousement leur partition secrète et sont moins proches d'une interprétation symbolique, moins clairement ouverts vers ce mouvement de s'habiter psychiquement, de s'approprier de soi. La fleur de papier japonais qui se déploie dans l'eau a parfois du mal à percer la carapace des résistances pour s'autoriser à fleurir avec bonheur. Ainsi en est-il de ce rêve de presque fin d'analyse, ou d'un arrêt d'analyse qui se continuera en auto-analyse. Il s'agit d'un rêve de «Dolarama». Do-la-ra-ma. Déjà la sonorité du mot l'apparente à la douleur. Et l'oriente rapidement vers une certaine pauvreté. La couleur du rêve est sombre; n'y perce aucune luminosité. L'affect est d'angoisse. Le récit mène à un échec :

«Un auditorium comme il s'en trouve dans les universités, ou encore, peut-être, est-ce une salle de thêâtre avec ses gradins. Il y règne une grande obscurité. Nous sommes au Dolarama. Je m'y trouve avec un homme. J'écoute une conférence. Ou est-ce moi qui donne la conférence ? Puis, je m'aperçois que j' ai perdu un bouton de ma veste. Cela m'embête beaucoup. C'est un bouton rouge que j'ai fait poser sur une veste de la même couleur, rouge. Des boutons fragiles et rares, italiens peut-être. Je veux chercher le bouton perdu et le retrouver. Il a dû tomber et rouler par terre. L'homme, à mes côtés, ne m'aide pas du tout, indifférent. Je déambule, dans le noir, parmi les rangées de sièges, un peu perdue. Je ne trouve rien. Je suis angoissée. Je ne le retrouverai pas.» 
J'ai perdu un bouton et c'est moi qui me retrouve perdue. Pourquoi ce décor de Do-la-ra-ma, cette obscurité, cette quasi-odeur de pauvreté qui me rappelle celle de ces magasins à un dollar? Pourquoi ce Dolarama que je ne cesse de prononcer Dolo-ra-ma, inscrivant le mot douleur au centre du rêve? L'intensité de la douleur m'est familière. Son frayage crée une tentation constante, héritage d'un univers maternel et féminin que l'analyse m'a fait reconnaître et m'invite à quitter. L'homme du rêve - est-ce l'analyste? - ne m'aide pas, répétant ainsi la détresse de l'enfant laissée seule avec sa peine, face à une mère indifférente. Où serait la réalisation du désir dans cette scène marquée par la répétition de la détresse, par la solitude ressentie de façon douloureuse au cour du groupe, par le désaide, par l'absence d'objet secourable et par l'échec ? Comment se faufilerait ici la trace du souhait réalisé dans le noirâtre des gradins où je me perds en perdant ce petit accessoire vestimentaire, cet accessoire luxueux, rouge, qui sait habiller le corps et le mettre au monde? La perte des objets est un thème connu. On peut ${ }^{3}$ mettre en relation les pertes d'objets à ce non-attachement que l'enfant a pu ressentir. On ne peut s'attacher à des objets si l'on n'a pas été, soi-même, investi. On ne peut qu'être tenté d'abandonner si l'on a été abandonné. Le non-soin ou l'indifférence reçus, perçus, vécus, se contaminent à soi comme aux annexes de soi. Mais cela n'empêche pas le regret qui suit la perte des objets négligés ou égarés. Souvent les rêves mettent en scène la perte des objets tels les sacs, les portefeuilles, les clés: objets liés à l'identité. Dans ce rêve, il s'agit d'un objet de luxe, dans son italianité, dans sa rareté: c'est l'envers même du décor de ce magasin à un dollar avec son odeur fétide. Je glisse de la pauvreté à la richesse, à mon goût de richesse. L'inversion est patente.

En restant un petit moment sur la condensation que représente le bouton, il me revient, avec cette précieuse méthode d'association qui, seule, permet que le rêve se déplie, le rappel d'une phobie de Marie Bonaparte : la phobie des boutons. Déjà, les initiales de Marie Bonaparte se tracent, cachées dans une identification glorieuse dont le rêve est si souvent porteur, narcissisme infantile oblige, comme un chiffre brodé sur l'étoffe du rêve. Et puis, j'ai fait ce rêve au moment où j'accomplissais des démarches pour le groupe d'analystes laïques dont je fais partie. Marie Bonaparte a été, je me le suis rappelé furtivement quelques jours auparavant, une ardente défenseur des psychanalystes laïques, dont elle faisait, elle aussi, partie. C'est pour elle et pour sa fille Anna que Freud écrivit en 1926, le texte sur l'analyse laïque. J'ai eu du mal à retrouver chez Marie Bonaparte cette référence au bouton; la voici, prise dans un chapitre du tome II de ses Mémoires, chapitre intitulé «Gloire et détresse de l'âme adolescente» :

«Je ne suis pas jolie. J'ai grandi trop vite : à quatorze ans, je suis presque aussi grande que Bonne-Maman, qui a un mètre soixante-dix. Je ne sais quoi faire de mes membres, qui pendent disgracieux autour de moi. J'ai souvent mal dans le dos et me courbe. [...] Et mon teint est plus jaune que jamais avec de l'acné. [...] Seuls mes yeux restent beaux, grands, d'un brun 
profond, surmontés de deux sourcils bien arqués et bien fournis. C'est quand je suis seule et nue dans ma chambre que je suis le moins mal. Les vêtements dont Bonne-Maman m'affuble doivent contribuer à ma disgrâce, comme aussi la coiffure plate qu'elle m'impose [...]. Mais surtout, surtout fierté nouvelle, mes seins poussent et se renflent, larges, avec des bouts délicats, de droite à gauche, sur mes côtés. Et une nouvelle chevelure frisée est venue orner le bas de mon corps, comme en compensation de mes cheveux trop plats qui tombent et vont se clairsemant.

Pourtant ces quelques adjuvants à ma nudité ne suffisent pas à m'inspirer la fierté de mon corps, de mon corps de femme. Je préférerais sans doute être un homme, et voir pousser à mon corps autre chose que des seins. C'est aussi pourquoi, en protestation aux boutons de mes seins, je ne peux plus voir sans une phobique horreur les boutons aux vêtements des femmes et des hommes. Non, je n'ai pas de prétention à la beauté, à cette beauté féminine en moi trop imparfaite.» [...] (Bonaparte, 1958, 552-553)

J'ai lu Marie Bonaparte, Derrière les vitres closes et L'appel des sèves, tous deux À la mémoire des disparus, en découpant les pages de ces livres empruntés à la bibliothèque de la Société psychanalytique de Montréal. C'est dire que peu de lecteurs s'y aventurent ! Il y a dans ces deux gros livres une naïveté dans l'expression écrite qui peut en arrêter plusieurs. S'y dévoile l'histoire d'une petite fille de la fin du XIX ${ }^{e}$ siècle, dont la mère meurt peu de jours après sa naissance. Cette mort, cette perte, fera naître en elle, dans le foisonnement intense d'une vie fantasmatique, toute une série de mythes magico-sexuels autour des énigmes de la vie et de la mort de même qu'un appétit très grand pour les criminels, voire les condamnés à mort, soudant ensemble meurtre et sexualité. C'est avec Marie Bonaparte et Sophie de Mijolla-Mellor qui l'a beaucoup lue que l'on comprend que, chez l'enfant, la mort, celle qui touche les êtres chers, porte toujours l'idée du meurtre — l'on ne meurt pas, on est tué ${ }^{3}$ — et que la sexualité apparaît nécessairement sur fond de violence et de sadisme infligés par un plus fort à un plus faible.

Marie Bonaparte, outre la Princesse qu'elle était de naissance, dans la lignée des Bonaparte, fut une petite fille seule, malade, mais surtout très seule; une petite fille qui entreprit très jeune une «vocation écrivassière », comme elle le disait ellemême. Ce besoin d'écrire l'a sans doute sauvée d'une mort psychique tout en liant, comme il arrive souvent, écriture et auto-érotisme dans une jouissance interdite et secrète, dans une excitation sans cesse relancée, sans cesse se nourrissant de son propre mouvement, avec le risque des inhibitions s'y afférant. Toute jeune, Marie Bonaparte écrit cinq petits Cahiers qu'elle appelle, comme le petit Hans, «Bêtises», sorte de journal intime consigné dans une langue qu'elle se fabrique mêlant, dans ses mots d'enfants, le français, l'anglais, l'allemand, pour bien s'assurer que sa grand-mère tellement haïe, cette Bonne-Maman qui la coiffe et 
l'habille si mal, ne pourra pas les lire. Cette écriture cherche à sceller le secret de ses rêveries tout en tentant de trouver un sens à tout ce qui lui arrive. Ces Cahiers sont-ils destinés à son père ? Oubliés, ils seront retrouvés dans la bibliothèque du père, après la mort de celui-ci, alors que Marie Bonaparte entreprend le rangement de la chambre du disparu. Elle les reconnaît à peine, elle s'y reconnaît à peine. Comme il arrive souvent que l'on ne reconnaisse pas de vieux rêves interprétés. Quand, peu de temps après cette mort, en 1925, la Princesse entreprendra une analyse avec Freud, elle lui apportera ces Cahiers pour qu'il puisse les interpréter. On peut regretter que Freud ne s'en soit pas servi pour reprendre ses théories sexuelles infantiles et ses théories de la féminité en devenir chez la petite fille. Ces Cahiers semblent receler, dans cette langue difficile à déchiffrer, des trésors inouïs sur les fantasmes infantiles et ceux, en particulier, des petites filles.

Grande rêveuse, Marie Bonaparte y écrivait aussi ses rêves. Certains, le cauchemar répétitif du Serquintuié, le Calica, ou le Chateigné ont été interprétés dans son analyse avec Freud. Ces rêves, pris dans le corps même de la sonorité des mots, renvoient à l'univers symbolique de Marie Bonaparte: le meurtre, le tué, le voyeurisme infantile, les scènes primitives dont l'enfant fut maintes fois témoin, le glissement de la nuque à l'anus, l'empoisonnement dont peut-être la mère fut victime, les images phalliques avec lesquelles la petite fille se débattait, comme ce «phallus cérébral» nécessaire pour accéder à la féminité ou en bloquer l'accès. Tout l'univers infantile de la petite princesse s'y retrouve dans sa nudité malgré le déguisement du travail du rêve. Pour beaucoup d'entre nous, le devenir-femme ne fut pas une chose aisée; il en fut de même pour Marie Bonaparte. Son activité d'écriture et son appétence envers la science la faisaient s'identifier à son père, chercheur et scientifique. Le travail intellectuel, lire et écrire par exemple, restera pour elle indexé au masculin. D'ailleurs, autour d'elle, sa grand-mère et son père ne l'incitent pas à étudier. C'est elle qui force le savoir, c'est elle qui rejette l'ignorance; c'est elle qui, curieuse, se passionne de tout, bien qu'elle ait ainsi l'impression de s'éloigner des vertus féminines. Elle lira Freud au chevet de son père. Ce sera, pour elle, une révélation. Elle y écrit aussi beaucoup dont «Monologues devant la vie et la mort », un écrit comportant de très belles réflexions d'une fille, d'une femme placée dans l'anticipation du deuil de son père.

Ce n'est qu'après la mort de son père qu'elle demande à Laforgue, qu'elle a rencontré à quelques reprises pour ses angoisses, de la présenter à Freud. Avec Freud, elle commencera une analyse en 1925, analyse entrecoupée de va-et-vient entre Vienne, Paris, la Grèce; analyse scandée d'interventions chirurgicales qui marqueront pour longtemps l'imaginaire féminin. Les opérations au clitoris - petit bouton rouge, s'il en est - ont commencé avant l'analyse de Marie Bonaparte avec Freud. Elles ont continué pendant son analyse. L'agir chirurgical ne s'est pas estompé avec l'élaboration psychique. Le recours au physiologique, à l'organique, restera, chez elle, une tentation constante. Objet phobique, objet aimé, faut-il voir, chez Marie Bonaparte, sous la phobie des boutons, énoncée à quatorze ans, un lien avec la place du clitoris dans la jouissance féminine? Dans l'extrait 
cité plus haut, elle semblait lier les boutons de ses seins aux boutons des vêtements: peut-on faire glisser l'association des boutons jusqu'au clitoris? Nata Minor note la très grande importance du clitoris dans les théories sexuelles de Marie Bonaparte. Au cours de son analyse avec Freud, Marie Bonaparte sentit le besoin de confirmer les constructions de son analyste quant aux scènes primitives dont elle aurait été témoin dans son enfance; elle alla vérifier le tout dans la réalité et questionna le vieux Pascal, le demi-frère de son père, qui avouera avoir souvent caressé, devant l'enfant, la nounou Mimau qui se serait écriée : «Ah ce bouton, ce bouton! » Nata Minor poursuit:

« Nous voici donc à l'orée d'une triste adolescence où le bouton fera la loi, s'imposant soudain, gâchant tous les plaisirs, tyrannique, obscène. [...] Objet aimé, le bouton et ses trous s'avère comme le héros d'un cauchemar qu'elle cherche à conjurer.» (Bourgeron, 1997, p. 57-58)

Conjurer le cauchemar, c'est ce que les opérations de déplacement du clitoris tenteront vainement de faire. Les rapprochements chirurgicaux clitoris-vagin n'arriveront jamais à sortir Marie Bonaparte de l'obsession de sa frigidité ni à lui donner, à travers maintes histoires d'amour, la jouissance demandée. Elle finira ses jours, fataliste, désabusée, posant sur elle-même un regard sans complaisance de «vie de femme ratée».

Ai-je négligé, à la fin, mon rêve de Do-la-ra-ma, l'auditorium et le bouton perdu ? Le mot renvoie à des prénoms féminins : Dora, Laura, Mara, prises dans le devenirfemme. L'ombre du rêve reste tracée en filigrane dans l'évocation de la sexualité féminine liée à la douleur. Par ailleurs, ce rêve m'a menée jusqu'à la princesse, qu'enfant, j'ai sûrement rêvé d'être. Que j'aurais aimé voir apparaitre près de moi, que j'aurais aimé rencontrer à travers ma mère ou mes grands-mères. Il m'est arrivé, dernièrement, de côtoyer une petite fille. Avec elle, dans ses grands yeux exigeants, j'ai appris que les petites filles non seulement aiment être des princesses, porter des robes de princesses, lire des histoires de princesses, voir des films de princesses, mais qu'elles ont aussi grand besoin que leur mère ou que leur grand-mère soient des princesses. C'est ainsi que, pour les petites filles, la chance de devenir princesse se cristallise. De plus, au même moment, elles découvrent que les sorcières créent plus de fascination que les princesses puisqu'elles brouillent à jamais les repères entre le bon et le mauvais, entre ce qu'il convient de haïr et ce qu'il convient d'aimer, entre celles qu'elles voudraient devenir et les traîtres sublimes, les ogres, qu'il leur faudra combattre. Brouiller les frontières, estomper les formes, renverser les rôles, échanger les masques, c'est aussi le jeu ou le travail du rêve, pourvu qu'il soit rendu possible.

\section{L'attrait du rêve}

Objet de nostalgie, dit Pontalis, le rêve nous renvoie à la mère, à l'incontournable corps de la mère. Comment ne pas comprendre et même céder à «l'attrait du 
rêve», tout comme à son mystère? Puisque son ombilic, ce qui nous relie à la mère, précisément, reste inaccessible. En même temps qu'il réveille l'infantile et ses tourments, le rêve est cet «objet perdu que nous ne voudrions plus perdre», objet précieux dont nous tentons de sauvegarder des bribes. Pourtant, interpréter un rêve, c'est s'arracher à l'envoûtement qu'il a produit, à sa fascination pour entrer dans une autre forme de fascination, celle de la parole ou de l'écriture. Interpréter, c'est sortir le rêve de l'immortalité et de la toute puissance qui l'irriguent; c'est fracasser l'unité de l'objet et du désir, c'est risquer de tuer le rêve. Voilà pourquoi tant de rêves retournent à leur totalité, évitant d'être fragmentés ou triturés par un psychanalyste déchiffreur d'énigmes. Événement de la nuit, le rêve est pensée du nocturne et parole d'enfant, d'un infans par ailleurs privé de parole mais tout en cris, en pleurs, en plaisirs, en désirs, en détresse. En faisant de son interprétation la voie royale vers l'inconscient, Freud arrache le rêve à sa nuit, à son intimité secrète et lui donne ce statut de pensée que la rationalité peine à lui reconnaître. Monde à part, hors d'une logique et d'une temporalité linéaire, le rêve communique avec l'obscur, avec l'infini: issu du puits noir de l'inconscient, il témoigne de la mobilité incessante de la psyché du dormeur. Le dormeur n'arrête pas de penser: il rêve. Et ces pensées resurgissent au réveil, dans leur bizarrerie, dans leur intensité, dans leur évanescence aussi.

«Qu'appelle-t-on pensée?» se demandait le philosophe Heidegger. Pour lui, comme pour le Freud de la «Psychopathologie de la vie quotidienne», la référence au poétique est nécessaire. Hölderlin ici, celui du «Nous sommes un Monstre, privé du sens », celui de la «Beauté», celui de «Qui le plus profond a pensé aime le plus vivant» indique que «le désir repose dans la pensée.» (Heidegger, 1959, 33) Avec le poète, nous ne sommes pas loin du rêve, mais alors sur quoi repose la pensée du rêve? Malgré son absence de logique, sa grammaire inconsciente, son travail de déformation, la pensée rêvante est-elle si étrangère à celle du diurne ? Penser le rêve, penser ce qu'il en est de la pensée du rêve, de son secret, de son étrangeté, de sa puissance, de son lien avec la sexualité infantile, serait, s'il faut suivre encore un peu le philosophe, quelque chose de «dangereux ${ }^{5} »$, de périlleux. C'est parler de son secret, de ses replis. On ne s'en approche pas sans en être transformé. Comme on ne fait pas l'expérience du rêve sans que la transformation, celle-là même que souhaite la psychanalyse, n'arrivât peu à peu à se réaliser.

Reviennent les mots de Philippe Forest placés au début de Sarinagara:

Tous les souvenirs enfin s'effacent. Et puis restent les rêves. Alors comme ils sont seuls désormais, c'est à eux que l'on confie le souci de sa vie.

marie claire lancôt bélanger

51 avenue nelson outremont québec h2v $3 \mathrm{z} 8$ canada courriel: mclb@aei.ca 
Filigrane, printemps 2007

\section{Références}

Anzieu, D., 1988, L'auto-analyse de Freud et la découverte de la psychanalyse, Paris, PUF.

Bonaparte, M., 1958, L'appel des Sèves, À la mémoire des disparus, tome II, Paris, PUF.

Bourgeron, J.-P., 1997, Marie Bonaparte, Paris, PUF, psychanalyse d'aujourd'hui.

de Mijolla-Mellor, S., 2002, Le besoin de savoir, théories et mythes magico-sexuels dans l'enfance, Paris, Dunod.

Dorra, M., 2005, Quelle petite phrase bouleversante au cœur d'un être ?, Paris, Gallimard, coll. Tracés.

Forest, P., 2004, Sarinagara, Paris, Gallimard, NRF.

Freud, S., 1900, L'interprétation des rêves, Paris, PUF, 1967.

Freud, S., 1901, Psychopathologie de la vie quotidienne, trad. Jankélévitch, Paris, Petite bibliothèque Payot, 2001.

Freud, S., 1920, Au-delà du principe du plaisir », in Essais de psychanalyse, Paris, Petite bibliothèque Payot.

Gribinski, M., 2005, Dialogue sur la nature du transfert, Paris, PUF, Petite bibliothèque de psychanalyse.

Guillaumin, J., 1979, Le rêve et le moi, Paris, PUF, Le fil rouge, 1979.

Heidegger, M., 1959, Qu'appelle-t-on penser ?, Paris, PUF, Épiméthée.

Lewin, B. D., 1949, Le sommeil, la bouche et l'écran du rêve, in Nouvelle Revue de psychanalyse, Paris, Gallimard, 1987.

Masud R. Khan, M., 1987, La capacité de rêver, in Nouvelle Revue de psychanalyse, Paris, Gallimard.

Perelberg, R. J., 2000, Jeux d'indentification, in Libres cahiers pour la psychanalyse, Paris, ${ }^{\circ} 1$, In Press,

Pontalis, J.-B., 1987, Perdre de vue, in Nouvelle revue de psychanalyse, Paris, Gallimard.

Pontalis, J,-B., 1990, La force d'attraction, Paris, Seuil.

Pontalis, J.-B., 2001, Le rêve à la source de la pensée ?, in Starobinski en mouvement, direction M. Gagnebin et C. Savinel, Paris, Champ Vallon.

Stevenson, R. L., 1888, Un chapitre sur les rêves, in L'étrange cas du docteur Jekyll et de M. Hyde, Gallimard, 2003.

Stirner, G., 1993, La mort de la tragédie, Paris, Gallimard, Folio essais.

Weiss, P., 1962, Schiller-Theater de Berlin.

\section{Notes}

1. Jean-Paul, dans son traité de 1798: «Le rêve n'est rien d'autre que poésie involontaire.»

2. Je pense au rêve qui ouvre le Sarinagara de Philippe Forest (2004); puis, à Vermeer et à Bergotte, bien sûr.

3. Rosine Jozef Perelberg (2002): «Anna Freud décrit comment les enfants perdent leurs affaires de façon chronique quand ils ne se sentent pas aimés. Ils sont incapables de s'approprier les choses ou de maintenir un lien de propriété avec elles.»

4. Idée déjà présente chez Freud (1920) : «car l'idée de la "mort naturelle" est étrangère aux peuples primitifs qui attribuent la mort de chacun d'entre eux à l'influence d'un ennemi ou d'un méchant esprit. »

5. «Mais il ne faut pas exclure non plus que ce qui se donne le plus à penser, précisément s'il est le plus haut, demeure en même temps le plus dangereux. Ou bien croit-on qu'un homme endurerait l'être du vrai, l'être du beau, l'être de la grâce, pour ne nommer qu'eux, tout simplement sans le moindre danger?» (Ibid., 37) 